Published By Institute of Physics Publishing for SISSA/ISAS

RECEIVED: August 13, 2003

ACCEPTED: October 14, 2003

\title{
Topological Poisson sigma models on Poisson-Lie groups
}

\author{
Iván Calvo, Fernando Falceto and David García-Álvarez \\ Departamento Física Teórica, Univ. Zaragoza \\ E-50009 Zaragoza, Spain \\ E-mail: icalvo@unizar.es, falceto@unizar.es, dga@saturno.unizar.es
}

\begin{abstract}
We solve the topological Poisson Sigma model for a Poisson-Lie group $G$ and its dual $G^{*}$. We show that the gauge symmetry for each model is given by its dual group that acts by dressing transformations on the target. The resolution of both models in the open geometry reveals that there exists a map from the reduced phase space of each model $\left(P\right.$ and $\left.P^{*}\right)$ to the main symplectic leaf of the Heisenberg double $\left(D_{0}\right)$ such that the symplectic forms on $P, P^{*}$ are obtained as the pull-back by those maps of the symplectic structure on $D_{0}$. This uncovers a duality between $P$ and $P^{*}$ under the exchange of bulk degrees of freedom of one model with boundary degrees of freedom of the other one. We finally solve the Poisson Sigma model for the Poisson structure on $G$ given by a pair of $r$-matrices that generalizes the Poisson-Lie case. The hamiltonian analysis of the theory requires the introduction of a deformation of the Heisenberg double.
\end{abstract}

Keywords: Topological Field Theories, Duality in Gauge Field Theories, Gaug Symmetry, Quantum Groups. 


\section{Contents}

1. Introduction 1

2. Poisson Sigma models 2

3. Poisson-Lie groups

4. Poisson-Lie Sigma model on $G$

5. Poisson-Lie Sigma model on $G^{*} \quad 8$

6. More general Poisson Sigma models on $G \quad 10$

7. Conclusions 12

\section{Introduction}

Since their appearance in [16, 13] the Poisson Sigma models have played a central role in the study of two dimensional gauge theories. They are related to pure gravity, to WessZumino-Witten models and Yang-Mills in two dimensions. Generalizations of the model that include supergravity have been recently proposed in [9, 5].

The Poisson Sigma models are topological and besides the two dimensional (spacetime) manifold $\Sigma$ all we require is a Poisson structure on the target $(M,\{\}$,$) (supplemented$ by boundary conditions if $\partial \Sigma \neq \emptyset)$. The theory seems then suitable for the study of the underlying Poisson structure and in fact in [7] it was shown that the semiclassical expansion of these models (with $\Sigma$ the unit disk and insertions at the boundary) reproduces the *-product introduced by Kontsevich in [14] that quantizes by deformation the Poisson bracket.

In this paper we shall be interested in Poisson Sigma models in which $M$ is a Lie group. In this case it is natural to ask for a consistency relation between its Poisson and group structures and this leads to the concept of Poisson-Lie group (17, 15). Poisson-Lie groups are the classical counterparts of quantum groups and they lead to the so called hidden symmetries of integrable systems that induce dressing transformations in the phase space. We shall give later a more detailed description of these objects, but for the moment we would like to stress that Poisson-Lie groups come in dual pairs under the exchange of the roles of Poisson and Lie brackets. Examples of Poisson-Lie Sigma models have been studied in [10, 4] in connection to $G / G$ Wess-Zumino-Witten theories. Here we pursue a systematic study of the matter and besides we shall also consider some generalizations of the Poisson structure for which the product with the corresponding Poisson-Lie group acts as a Poisson action. 
One of the simplest examples of Poisson-Lie Sigma models is the linear one. Here $M$ is a vector space (abelian group) and its Poisson structure is linear. This model is related to $B F$ and Yang-Mills and can be considered as dual of the trivial Poisson-Lie Sigma model in an (in general) non-abelian group with vanishing Poisson bracket. Our models can be regarded as the simplest generalizations of the linear ones with which they share some properties that will be stressed in the sequel.

Another aspect that will deserve our attention is that of duality, i.e. we shall try to relate the two dual models, which is not obvious at the lagrangian level. However, in the hamiltonian approach, in the open geometry, the duality becomes evident: it consists of the exchange of bulk and boundary degrees of freedom. We would like to relate this fact with the non-abelian T-duality for WZW Sigma models ([2]).

We begin with a brief review of the essential results about Poisson Sigma models and Poisson-Lie groups in sections 2 and 3 . Sections 1 and 5 are devoted to the study of the Poisson-Lie Sigma model of a simple Poisson-Lie group and its dual one, respectively. By using the techniques of the two previous sections we are able to solve more general Poisson Sigma models on Lie groups and we introduce a generalization of the Heisenberg double in section 6. The conclusions and open questions are presented in section 7 .

\section{Poisson Sigma models}

The Poisson Sigma model is a two-dimensional topological Sigma model with the target manifold $M$ equipped with a Poisson structure $\Gamma \in T^{\wedge 2}(M)$, i.e. a bivector field on $M$. The Poisson bracket of two functions on $M$ is given by the contraction of $\Gamma:\{f, g\}(m)=$ $\iota\left(\Gamma_{m}\right) d f \wedge d g$.

The fields of the model are $X: \Sigma \rightarrow M$ and a 1-form $\psi$ on $\Sigma$ with values in the pullback by $X$ of the cotangent bundle of $M$. The action functional has the form

$$
S_{P \sigma}(X, \psi)=\int_{\Sigma}\langle d X, \wedge \psi\rangle-\frac{1}{2}\langle\Gamma \circ X, \psi \wedge \psi\rangle
$$

where $\langle$,$\rangle denotes the pairing between the tangent and the cotangent vectors to M$.

If $X^{i}$ are local coordinates on $M, \sigma^{\mu}$ local coordinates on $\Sigma, \Gamma^{i j}$ the components of the Poisson structure in these coordinates and $\psi_{i}=\psi_{i \mu} d \sigma^{\mu}, i=1, \ldots, n, \mu=1,2$ the action reads

$$
S_{P \sigma}(X, \psi)=\int_{\Sigma} d X^{i} \wedge \psi_{i}-\frac{1}{2} \Gamma^{i j}(X) \psi_{i} \wedge \psi_{j}
$$

It is straightforward to work out the equations of motion:

$$
\begin{aligned}
d X^{i}+\Gamma^{i j}(X) \psi_{j} & =0 \\
d \psi_{i}+\frac{1}{2} \partial_{i} \Gamma^{j k}(X) \psi_{j} \wedge \psi_{k} & =0
\end{aligned}
$$

One can show ([6]) that for solutions of (2.3a) the image of $X$ lies within one of the symplectic leaves of the foliation of $M$. 
Under the infinitesimal transformation

$$
\begin{aligned}
\delta_{\epsilon} X^{i} & =\epsilon_{j} \Gamma^{j i}(X) \\
\delta_{\epsilon} \psi_{i} & =d \epsilon_{i}+\partial_{i} \Gamma^{j k}(X) \psi_{j} \epsilon_{k}
\end{aligned}
$$

where $\epsilon=\epsilon_{i} d X^{i}$ is a section of $X^{*}\left(T^{*}(M)\right)$, the action (2.2) transforms by a boundary term

$$
\delta_{\epsilon} S_{P \sigma}=-\int_{\Sigma} d\left(d X^{i} \epsilon_{i}\right)
$$

One of the special properties of these models is that the commutator of two consecutive gauge tansformation of (2.4) is not of the same form i.e.

$$
\begin{aligned}
{\left[\delta_{\epsilon}, \delta_{\epsilon^{\prime}}\right] X^{i} } & =\delta_{\left[\epsilon, \epsilon^{\prime}\right]^{*}} X^{i} \\
{\left[\delta_{\epsilon}, \delta_{\epsilon^{\prime}}\right] \psi_{i} } & =\delta_{\left[\epsilon, \epsilon^{\prime}\right]^{*}} \psi_{i}+\epsilon_{k} \epsilon_{l}^{\prime} \partial_{i} \partial_{j} \Gamma^{k l}\left(d X^{j}+\Gamma^{j s}(X) \psi_{s}\right)
\end{aligned}
$$

where for a vector field $\chi \in \mathfrak{X}(M), \iota(\chi)\left[\epsilon, \epsilon^{\prime}\right]^{*}=\iota\left(\mathcal{L}_{\chi} \Gamma\right) \epsilon \wedge \epsilon^{\prime}$. Later, we shall make extensive use of this commutator in the context of Poisson-Lie groups. Note that the term in parenthesis in (2.6b) is the equation of motion (2.3a), and then it vanishes on-shell. However, as remarked in [6], due to the $X$ dependence of $\left[\epsilon, \epsilon^{\prime}\right]^{*}$ even on-shell one can not talk properly about a Lie algebra structure in the space of parameters, unless we enlarge its definition to account for field dependent gauge transformations. The situation will become simpler in the linear case that we describe below.

One of the simplest examples of Poisson Sigma models is the linear one (2D BF theory). We take a finite dimensional vector space $V$ as the target space and define a linear Poisson bracket on it, i.e. for any $\phi, \psi \in V^{*}$, their Poisson bracket $\{\phi, \psi\} \in V^{*}$. The linear Poisson bracket on $V$ defines then a Lie bracket [, ] on $V^{*} \equiv \mathfrak{g}$. Assuming that $\mathfrak{g}$ is semisimple we can use the Cartan-Killing form tr to identify $\mathfrak{g}$ and $\mathfrak{g}^{*}$. The fields are then $X \in \Lambda^{0}(\Sigma) \otimes \mathfrak{g}$ and $A \in \Lambda^{1}(\Sigma) \otimes \mathfrak{g}$ and the action reads:

$$
S_{l}(X, A)=\int_{\Sigma} \operatorname{tr}\left(d X \wedge A+\frac{1}{2} A \wedge[X, A]\right)
$$

The equations of motion are:

$$
\begin{aligned}
d A+[A, A] & =0 \\
d X+[A, X] & =0
\end{aligned}
$$

For $\epsilon \in \Lambda^{0}(M) \otimes \mathfrak{g}$ the gauge transformation

$$
\begin{aligned}
\delta_{\epsilon} X & =[X, \epsilon] \\
\delta_{\epsilon} A & =d \epsilon+[A, \epsilon]
\end{aligned}
$$

induces the change of the action (2.7) by a boundary term

$$
\delta_{\epsilon} S_{l}=-\int_{\Sigma} d \operatorname{tr}(d X \epsilon)
$$

Note that in this case the gauge transformations close even off-shell

$$
\left[\delta_{\epsilon}, \delta_{\epsilon^{\prime}}\right]=\delta_{\left[\epsilon, \epsilon^{\prime}\right]}
$$

and induce the Lie algebra structure of $\Lambda^{0}(\Sigma) \otimes \mathfrak{g}$ in the space of parameters. 


\section{Poisson-Lie groups}

In this section we shall review some basic features of the theory of Poisson-Lie groups and fix our notation. See [17, 10, 15, 3, 11] for details. A Poisson-Lie group is a Lie group equipped with a Poisson structure which makes the product $m: G \times G \rightarrow G$ a Poisson map if $G \times G$ is considered with the product Poisson structure. Linearization of the Poisson structure at the unit $e$ of $G$ provides a Lie algebra structure on $\mathfrak{g}^{*}=T_{e}^{*}(G)$ by the formula

$$
\left[d \xi_{1}(e), d \xi_{2}(e)\right]^{*}=d\left\{\xi_{1}, \xi_{2}\right\}(e)
$$

The Poisson-Lie structure of $G$ yields the compatibility condition

$$
\left\langle[X, Y],[v, w]^{*}\right\rangle+\left\langle\operatorname{ad}_{v}^{*} X, \operatorname{ad}_{Y}^{*} w\right\rangle-\left\langle\operatorname{ad}_{w}^{*} X, \operatorname{ad}_{Y}^{*} v\right\rangle-\left\langle\operatorname{ad}_{v}^{*} Y, \operatorname{ad}_{X}^{*} w\right\rangle+\left\langle\operatorname{ad}_{w}^{*} Y, \operatorname{ad}_{X}^{*} v\right\rangle=0 .
$$

which allows to define a Lie bracket in $\mathfrak{g} \oplus \mathfrak{g}^{*}$ by the formula

$$
[X+\xi, Y+\zeta]=[X, Y]+[\xi, \zeta]^{*}-a d_{X}^{*} \zeta+a d_{Y}^{*} \xi+a d_{\zeta}^{*} X-a d_{\xi}^{*} Y
$$

If $G$ is connected and simply connected, (3.2) is enough to integrate [ , ]* to a Poisson structure on $G$ that makes it Poisson-Lie and the Poisson structure is unique. The symmetry between $\mathfrak{g}$ and $\mathfrak{g}^{*}$ in (3.2), implies that one has also a Poisson-Lie group $G^{*}$ with Lie algebra $\left(\mathfrak{g}^{*},[,]^{*}\right)$ and a Poisson structure whose linearization at $e$ gives the bracket $[$,$] .$ $G^{*}$ is the dual Poisson-Lie group of $G$.

Let us take $G$ a complex, simple, connected, simply connected Lie group and give the above construction explicitly. The (essentially unique) nondegenerate, invariant, bilinear form $\operatorname{tr}$ on $\mathfrak{g}$ establishes an isomorphism between $\mathfrak{g}$ and $\mathfrak{g}^{*}$. The Poisson structure $\Gamma$ contracted with the right-invariant forms $\Lambda(X)=\operatorname{tr}\left(d g g^{-1} X\right), X \in \mathfrak{g}$, will be denoted

$$
\gamma_{g}(X, Y)=\iota\left(\Gamma_{g}\right) \Lambda(X) \wedge \Lambda(Y)
$$

For a general Poisson-Lie structure on $G(15])$,

$$
\gamma_{g}^{r}(X, Y)=\frac{1}{2} \operatorname{tr}\left(X r Y-X A d_{g} r A d_{g}^{-1} Y\right)
$$

where $r: \mathfrak{g} \rightarrow \mathfrak{g}$ is an antisymmetric linear operator such that

$$
r[r X, Y]+r[X, r Y]-[r X, r Y]=\alpha[X, Y], \quad \alpha \in \mathbb{C}
$$

Using the operator $r$ it is possible to define a new Lie bracket in $\mathfrak{g}$,

$$
[X, Y]_{r}=\frac{1}{2}([X, r Y]+[r X, Y])
$$

$\left(\mathfrak{g},[,]_{r}\right)$ is isomorphic to $\left(\mathfrak{g}^{*},[,]^{*}\right)$ via tr.

If $\alpha \neq 0$ we can rescale the bilinear form in $\mathfrak{g}$ and $r$ so that we leave the Poisson bracket unchanged but $\alpha=1$. In the following we shall consider this case $(\alpha=1)$ that

corresponds to the factorizable Lie bialgebras of ref. [18]. Take $r_{ \pm}=\frac{1}{2}(r \pm I), \mathfrak{d}=\mathfrak{g} \oplus \mathfrak{g}$ and $[,]_{\mathfrak{d}}=([],,[]$,$) . Then,$

$$
\left[r_{ \pm} X, r_{ \pm} Y\right]=r_{ \pm}[X, Y]_{r}
$$


and the embedding $\sigma: \mathfrak{g} \rightarrow \mathfrak{d}, X \mapsto\left(r_{+} X, r_{-} X\right)$ defines an homomorphism from $\left(\mathfrak{g},[,]_{r}\right)$ to $(\mathfrak{d},[,] \mathfrak{d})$. We give an invariant, nondegenerate bilinear form on $\mathfrak{d}$ by

$$
\operatorname{Tr}\left(\left(X_{1}, X_{2}\right)\left(Y_{1}, Y_{2}\right)\right)=\operatorname{tr}\left(X_{1} Y_{1}\right)-\operatorname{tr}\left(X_{2} Y_{2}\right)
$$

This allows to identify $\left(\mathfrak{g}^{*},[,]^{*}\right)$ with the subalgebra $\mathfrak{g}_{r}:=\sigma(\mathfrak{g}) \subset \mathfrak{d}$ and $G^{*}$ as the subgroup $G_{r}$ in $D:=G \times G$ corresponding to the Lie subalgebra $\mathfrak{g}_{r}$. We denote by $\left(g_{+}, g_{-}\right)$the elements of $G_{r}$. In a natural way, $\mathfrak{g} \cong \mathfrak{g}_{d}:=\{(X, X) \in \mathfrak{d} \mid X \in \mathfrak{g}\}$ and $G \cong G_{d}:=\{(g, g) \in D \mid g \in G\}$. Notice that $\mathfrak{g}_{d} \bigcap \mathfrak{g}_{r}=0 \Rightarrow G_{0}:=G_{d} \cap G_{r}$ is a discrete subgroup of $G$.

The induced Poisson-Lie structure on $G_{r}$ contracted with the right-invariant forms on $G_{r}, \Lambda^{r}(X)=\operatorname{tr}\left[\left(d g_{+} g_{+}^{-1}-d g_{-} g_{-}^{-1}\right) X\right]$ for $X \in \mathfrak{g}$, takes the form

$$
\gamma_{\left(g_{+}, g_{-}\right)}^{r}(X, Y)=\operatorname{tr}\left[X\left(A d_{g_{+}}-A d_{g_{-}}\right)\left(r_{-} A d_{g_{+}}^{-1}-r_{+} A d_{g_{-}}^{-1}\right) Y\right] .
$$

The $r$-matrices are used not only to give explicit realizations of the dual group and to construct Poisson-Lie structures but to construct more general Poisson structures on Lie groups. Following Semenov-Tian-Shansky (17]), if $r$ and $r^{\prime}$ are antisymmetric and satisfy (3.5) with the same value for $\alpha$, we can define the Poisson structure (contracted with the right-invariant forms),

$$
\gamma_{g}^{r, r^{\prime}}(X, Y)=\frac{1}{2} \operatorname{tr}\left(X r Y+X A d_{g} r^{\prime} A d_{g}^{-1} Y\right)
$$

$G$ equipped with 3.10 is denoted by $G_{r, r^{\prime}}$. One can verify that $G_{r,-r^{\prime}} \times G_{r^{\prime}, r^{\prime \prime}} \rightarrow G_{r, r^{\prime \prime}}$ is a Poisson map. When $r^{\prime}=r$ and $r^{\prime \prime}=-r$ then we get (3.4) and the Poisson structure is, indeed, Poisson-Lie.

Given a $r$-matrix in $G$ it is possible to define a $r$-matrix in $D=G \times G$ (which we shall denote by $R$ ) in a natural way: $R=P_{d}-P_{r}$ where $P_{d}$ and $P_{r}$ are the projectors on $\mathfrak{g}$ and $\mathfrak{g}_{r}$ respectively, parallel to the complementary subalgebra. Hence, $R_{+}=P_{d}, R_{-}=-P_{r}$.

We shall need the description of the Heisenberg double (which is not a Poisson-Lie group; in fact, with the notation introduced before, it corresponds to $D_{R, R}$ ). Its main symplectic leaf is $D_{0}=G_{d} G_{r} \cap G_{r} G_{d}$ (that contains a neighborhood of the unit of $D$ ). The symplectic structure obtained by inverting the Poisson structure on it reads

$$
\Omega\left(\left(h_{+} g, h_{-} g\right)\right)=\operatorname{tr}\left[d \widetilde{g} \widetilde{g}^{-1} \wedge\left(d h_{+} h_{+}^{-1}-d h_{-} h_{-}^{-1}\right)+g^{-1} d g \wedge\left(\widetilde{h}_{+}^{-1} d \widetilde{h}_{+}-\widetilde{h}_{-}^{-1} d \widetilde{h}_{-}\right)\right]
$$

where $\left(h_{+} g, h_{-} g\right)=\left(\widetilde{g} \widetilde{h}_{+}, \widetilde{g} \widetilde{h}_{-}\right) \in D_{0}$. Note that although these decompositions are not unique, the ambiguity (the product by an element of $G_{0}$ ) does not affect the values of $\Omega$ which is well defined in $D_{0}$.

\section{Poisson-Lie Sigma model on $G$}

Recall the action (2.1) and take the Poisson-Lie group of the previous section as the target. The action for $g: \Sigma \rightarrow G$ and $A \in \Lambda^{1}(\Sigma) \otimes \mathfrak{g}$ reads:

$$
S_{\mathrm{PL}}(g, A)=\int_{\Sigma} \operatorname{tr}\left(d g g^{-1} \wedge A\right)-\frac{1}{4} \operatorname{tr}\left(A \wedge\left(r-A d_{g} r A d_{g}^{-1}\right) A\right)
$$

which is what we call the Poisson-Lie Sigma model for $G$. 
We proceed now to a detailed study of this model, from which we shall learn techniques applicable to the resolution of more general Poisson Sigma models on Lie groups.

The equations of motion are:

$$
\begin{aligned}
d g g^{-1}+\frac{1}{2}\left(r-A d_{g} r A d_{g}^{-1}\right) A & =0 \\
d \widetilde{A}+[\widetilde{A}, \widetilde{A}]_{r} & =0, \quad \widetilde{A}:=A d_{g}^{-1} A
\end{aligned}
$$

From the previous two equations one can derive also a zero curvature equation for $A$ itself

$$
d A+[A, A]_{r}=0
$$

which can be deduced directly from the stationary condition for the action under variations of the fields that keep $\widetilde{A}$ invariant.

The gauge symmetry of the action, for $\beta: \Sigma \rightarrow \mathfrak{g}$, is given in its infinitesimal form by

$$
\begin{aligned}
\delta_{\beta} g g^{-1} & =\frac{1}{2}\left(A d_{g} r A d_{g}^{-1}-r\right) \beta \\
\delta_{\beta} A & =d \beta+[A, \beta]_{r}-\frac{1}{2}\left[d g g^{-1}+\frac{1}{2}\left(r-A d_{g} r A d_{g}^{-1}\right) A, \beta\right] .
\end{aligned}
$$

Under this transformation the action changes by a boundary term, namely $\delta_{\beta} S_{\mathrm{PL}}=$ $\int_{\Sigma} d \operatorname{tr}\left(g d g^{-1} \beta\right)$. Note that (4.4) corresponds to the right dressing vector fields of ref. 15. translated to the origin by right multiplication in $G$. Its integration (local as in general the vector field is not complete) gives rise to the dressing transformation of $g$. Also in (4.4) the third term vanishes on-shell; it corresponds to a trivial transformation (see refs. [6, [12]), proportional to the equations of motion. If we forget about that term (or alternatively, working on-shell)

$$
\left[\delta_{\beta_{1}}, \delta_{\beta_{2}}\right]=\delta_{\left[\beta_{1}, \beta_{2}\right]_{r}} .
$$

Note that unlike the general case this relation defines a Lie algebra structure (that of the gauge group corresponding to $G_{r}$ ) in the space of gauge transformations. This is also what happens in the linear case discussed in section 2. However, in this case the relation holds uniquely on-shell while in the linear case the Lie algebra structure persists off-shell. Another difference is that the vector fields (on-shell) induced by the gauge transformation are not complete in the general case. This is a second obstruction to properly talk about a gauge group, so we shall restrict ourselves to infinitesimal gauge transformations.

Now we proceed to integrate the equations of motion. We shall consider $\alpha=1$ in eq. (3.5) although as remarked before the case $\alpha \neq 0$ can be treated similarly. As the equation of motion for $A$ is independent of $g$, like in the linear model, we first solve the eqs. (4.2) and (4.3). This is in contrast with the general case of ref. [6]. Locally

$$
\begin{aligned}
A= & h_{+} d h_{+}^{-1}-h_{-} d h_{-}^{-1} \\
\widetilde{A}= & \widetilde{h}_{+} d \widetilde{h}_{+}^{-1}-\widetilde{h}_{-} d \widetilde{h}_{-}^{-1} \\
& \left(h_{+}, h_{-}\right) \text {and }\left(\widetilde{h}_{+}, \widetilde{h}_{-}\right): U \rightarrow G_{r}, \quad \Sigma \supset U \text { open contractible. }
\end{aligned}
$$


In order to fix the ambiguity in the new fields we choose a point $\sigma_{0} \in U$ and take $h_{ \pm}\left(\sigma_{0}\right)=\widetilde{h}_{ \pm}\left(\sigma_{0}\right)=e$.

We need to work a bit more to get explicit solutions for $g$. The equation of motion (4.2) can be equivalently written as

$$
d g g^{-1}+\left(r_{+}-A d_{g} r_{+} A d_{g}^{-1}\right) A=0
$$

and recalling that $\widetilde{A}=A d_{g}^{-1} A$,

$$
d g g^{-1}+r_{+} A-A d_{g} r_{+} \widetilde{A}=0
$$

$r_{+}$projects on the + component of the elements of $\mathfrak{g}$ and yields

$$
d g g^{-1}+h_{+} d h_{+}^{-1}-A d_{g} \widetilde{h}_{+} d \widetilde{h}_{+}^{-1}=0
$$

which is equivalent to

$$
\widetilde{h}_{+}^{-1} g^{-1} h_{+} d\left(h_{+}^{-1} g \widetilde{h}_{+}\right)=0 \Rightarrow h_{+}^{-1} g \widetilde{h}_{+}=\widehat{g}, \quad \widehat{g}=g\left(\sigma_{0}\right) \in G
$$

The same procedure can be carried out by using $r_{-}$,

$$
h_{-}^{-1} g \widetilde{h}_{-}=\widehat{g}
$$

The solutions for the equations of motion can then be expressed by the single relation in $D$ :

$$
\left(g(\sigma) \widetilde{h}_{+}(\sigma), g(\sigma) \widetilde{h}_{-}(\sigma)\right)=\left(h_{+}(\sigma) \widehat{g}, h_{-}(\sigma) \widehat{g}\right)
$$

which in particular implies that $\left(h_{+}(\sigma) \widehat{g}, h_{-}(\sigma) \widehat{g}\right) \in D_{0}$, and $g(\sigma)$ takes values in the connected component of orbits of $\widehat{g}$ by dressing transformations. These orbits are the symplectic leaves of the Poisson-Lie group $G$.

Now we proceed to the hamiltonian analysis of the model. To that end we fix the open geometry for $\Sigma=[0, \pi] \times \mathbb{R}$ and take free boundary conditions for $g$ and $A$ vanishing on vectors tangent to $\partial \Sigma$. The boundary conditions enforce that $h_{ \pm}, \widetilde{h}_{ \pm}$are constant along the connected components of the boundary, $h_{ \pm}(0, t)=h_{0 \pm}, \widetilde{h}_{ \pm}(0, t)=\widetilde{h}_{0 \pm}, h_{ \pm}(\pi, t)=h_{\pi \pm}$, $\widetilde{h}_{ \pm}(\pi, t)=\widetilde{h}_{\pi \pm}$. The presymplectic form is

$$
\omega=\int_{\mathcal{C}} \operatorname{tr}\left(\delta g g^{-1} \wedge \delta g g^{-1} A-\delta g g^{-1} \wedge \delta A\right)
$$

where $g$ and $A$ varies in the space of solutions of the equations of motion and $\mathcal{C}$ is any curve joining the two components of the boundary. The result is, of course, independent of the particular choice of $\mathcal{C}$.

If we parametrize the solutions in terms of $h_{ \pm}(\sigma), \widehat{g}$ with $\left(h_{+}(\sigma) \widehat{g}, h_{-}(\sigma) \widehat{g}\right) \in D_{0}$ we obtain

$$
\omega=\frac{1}{2} \int_{\mathcal{C}} d \Omega\left(\left(h_{+}(\sigma) \widehat{g}, h_{-}(\sigma) \widehat{g}\right)\right)
$$

where $d$ acts on the space-time variables in $\Sigma$. Then $\omega$ depends only on the values at the boundaries, namely

$$
\omega=\frac{1}{2}\left[\Omega\left(\left(h_{\pi+} \widehat{g}, h_{\pi-} \widehat{g}\right)\right)-\Omega\left(\left(h_{0+} \widehat{g}, h_{0-} \widehat{g}\right)\right)\right]
$$


Or if we take $\sigma_{0}=\left(0, t_{0}\right)$, i.e. $h_{0 \pm}=\widetilde{h}_{0 \pm}=e$

$$
\omega=\frac{1}{2} \Omega\left(\left(h_{\pi+} \widehat{g}, h_{\pi-} \widehat{g}\right)\right) .
$$

From the analysis of the degeneracy of $\omega$ we conclude that the vector fields induced by infinitesimal gauge transformations that are the identity at the boundary form its kernel and we reduce the space of solutions by this transformations. The reduced phase space $P$ is then the set of pairs $\left(\left[\left(h_{+}, h_{-}\right)\right], \widehat{g}\right)$ with $\left[\left(h_{+}, h_{-}\right)\right]$a homotopy class of maps from $[0, \pi]$ to $G_{r}$ which are the identity at 0 and have fixed value at $\pi$ and such that $\left(h_{+}(x) \widehat{g}, h_{-}(x) \widehat{g}\right) \in$ $D_{0}, x \in[0, \pi]$. The symplectic form on $P$ can be viewed as the pullback of $\Omega$ by the map $\left(\left[h_{+}, h_{-}\right], \widehat{g}\right) \mapsto\left(h_{\pi+} \widehat{g}, h_{\pi-} \widehat{g}\right)$.

The set of solutions may be endowed with the structure of a symplectic groupoid ([7]). The product of two such solutions $\left(\left[\left(h_{+}, h_{-}\right)\right], \widehat{g}\right)$ and $\left(\left[\left(h_{+}^{\prime}, h_{-}^{\prime}\right)\right], \widehat{g}^{\prime}\right)$ is defined if $h_{\pi \pm} \widehat{g} \widetilde{h}_{\pi \pm}^{-1}=\widehat{g}^{\prime}$ and is given by $\left(\left[\left(h_{+}^{\prime \prime}, h_{-}^{\prime \prime}\right)\right], \widehat{g}\right)$ with

$$
h_{ \pm}^{\prime \prime}(x)= \begin{cases}h_{ \pm}(2 x) & 0 \leq x \leq \frac{1}{2} \pi \\ h^{\prime}(2 x-\pi)_{ \pm} h_{\pi \pm} & \frac{1}{2} \pi \leq x \leq \pi\end{cases}
$$

The results obtained in this section have their dual counterpart if we consider the dual group $G^{*}$, which is accomplished in the next section.

\section{Poisson-Lie Sigma model on $G^{*}$}

In order to unravel the role of the duality between Poisson-Lie groups in the context of Poisson-Lie Sigma models we proceed now to the study of the theory for $G^{*} \equiv G_{r}$. Using the Poisson structure given in (3.9), the action of the model reads,

$$
\begin{aligned}
S_{\mathrm{PL}}^{*}\left(g_{+}, g_{-}, A\right)=\int_{\Sigma} \operatorname{tr}[ & \left(d g_{+} g_{+}^{-1}-d g_{-} g_{-}^{-1}\right) \wedge A+ \\
+ & \left.\frac{1}{2} A \wedge\left(\operatorname{Ad}_{g_{+}}-\mathrm{Ad}_{g_{-}}\right)\left(r_{+} \operatorname{Ad}_{g_{-}}^{-1}-r_{-} \operatorname{Ad}_{g_{+}}^{-1}\right) A\right]
\end{aligned}
$$

As shown in [4, 10], this Poisson-Lie Sigma model with target $G_{r}$ and fields $\left(g_{+}, g_{-}\right)$ and $A$ is closely related to the $G / G$ gauged WZW model with fields $g=g_{-} g_{+}^{-1}$ and $A$. We do not pursue further this relation in the present work. We rather shall make a study analogous to that of the previous section, stressing its similarities and differences with respect to the Poisson-Lie Sigma model for $G$.

The equations of motion of the model are

$$
\begin{aligned}
g_{ \pm}^{-1} d g_{ \pm}+r_{ \pm}\left(A d_{g_{+}}^{-1}-A d_{g_{-}}^{-1}\right) A & =0 \\
d \widetilde{A}+[\widetilde{A}, \widetilde{A}] & =0 \\
\text { with } \widetilde{A}: & =\left(r_{+} A d_{g_{-}}^{-1}-r_{-} A d_{g_{+}}^{-1}\right) A .
\end{aligned}
$$


Note that, similarly to the previous case for the group $G$, the relation between $\widetilde{A}$ and $A$ is nothing but the coadjoint action of $\left(g_{+}, g_{-}\right)$for the group $G_{r}$. Like in the former case it is posible to deduce, from the previous equations, a zero curvature condition for $A$ itself

$$
d A+[A, A]=0 .
$$

The gauge transformations, for $\beta: \Sigma \rightarrow \mathfrak{g}$, read:

$$
\begin{aligned}
g_{ \pm}^{-1} \delta_{\beta} g_{ \pm}= & r_{ \pm}\left(A d_{g_{-}}^{-1}-A d_{g_{+}}^{-1}\right) \beta \\
\delta_{\beta} A= & d \beta+[A, \beta]+ \\
& +\frac{1}{2}\left(r_{+} A d_{g_{-}}+r_{-} A d_{g_{+}}\right)\left[g_{+}^{-1} d g_{+}-g_{-}^{-1} d g_{-}+\left(A d_{g_{+}}^{-1}-A d_{g_{-}}^{-1}\right) A, \widetilde{\beta}\right]
\end{aligned}
$$

where $\widetilde{\beta}:=\left(r_{+} A d_{g_{-}}^{-1}-r_{-} A d_{g_{+}}^{-1}\right) \beta$. The change in the action is $\delta_{\beta} S_{\mathrm{PL}}^{*}=\int_{\Sigma} d \operatorname{tr}\left(\left(g_{+} d g_{+}^{-1}-\right.\right.$ $\left.\left.g_{-} d g_{-}^{-1}\right) \beta\right)$. As before, if we forget about the third term on the right hand side of (5.4b), that vanishes on-shell, the transformations close. Namely $\left[\delta_{\beta}, \delta_{\gamma}\right]=\delta_{[\beta, \gamma]}$ which corresponds to the gauge group $G$.

The solutions of the equations of motion can be obtained along the same lines as before. From (5.3)

$$
A=h d h^{-1}
$$

in any contractible, open set. Without loss of generality we fix again a point $\sigma_{0} \in \Sigma$ such that $h\left(\sigma_{0}\right)=e$. Now we write together the equations for $g$ using the solution for $A$.

$$
g_{+}^{-1} d g_{+}-g_{-}^{-1} d g_{-}+\left(A d_{g_{+}}^{-1}-A d_{g_{-}}^{-1}\right) h d h^{-1}=0
$$

or equivalently

$$
h^{-1} g_{+} g_{-}^{-1} h d\left(h^{-1} g_{-} g_{+}^{-1} h\right)=0 \Rightarrow h^{-1} g_{-} g_{+}^{-1} h=\widehat{g}_{-} \widehat{g}_{+}^{-1}
$$

where $\widehat{g}_{ \pm}=g_{ \pm}\left(\sigma_{0}\right)$. Hence

$$
g_{+}^{-1} h \widehat{g}_{+}=g_{-}^{-1} h \widehat{g}_{-}=: \widetilde{h}
$$

and once more, we can obtain the solutions from an equation in $D$

$$
\left(g_{+}(\sigma) \widetilde{h}(\sigma), g_{-}(\sigma) \widetilde{h}(\sigma)\right)=\left(h(\sigma) \widehat{g}_{+}, h(\sigma) \widehat{g}_{-}\right),
$$

which means that $\left(g_{+}, g_{-}\right)$is the dressing-transformed of $\left(\widehat{g}_{+}, \widehat{g}_{-}\right)$by $h$. Orbits of the dressing transformation are the symplectic leaves of $G_{r}$. Note, at this point, the complete symmetry with the previous situation under the exchange of the roles of $G$ and $G_{r}$.

We consider again the open geometry $\Sigma=[0, \pi] \times \mathbb{R}$ with boundary conditions for $A$ vanishing on vectors tangent to the boundary and $g$ free. The boundary conditions impose that $h$ and $\widetilde{h}$ are constant in every connected component of $\partial \Sigma$ i.e. $h(0, t)=h_{0}$, $h(\pi, t)=h_{\pi}, \widetilde{h}(0, t)=\widetilde{h}_{0}, \widetilde{h}(\pi, t)=\widetilde{h}_{\pi}$.

The presymplectic form of the model $\omega^{*}$ can be written

$$
\omega^{*}=\frac{1}{2} \int_{\mathcal{C}} d \Omega\left(h(\sigma) \widehat{g}_{+}, h(\sigma) \widehat{g}_{-}\right)
$$


where again $d$ acts on the $\sigma$ variables. Therefore

$$
\omega^{*}=\frac{1}{2}\left[\Omega\left(\left(h_{\pi} \widehat{g}_{+}, h_{\pi} \widehat{g}_{-}\right)\right)-\Omega\left(\left(h_{0} \widehat{g}_{+}, h_{0} \widehat{g}_{-}\right)\right)\right]
$$

and taking $\sigma_{0}=\left(0, t_{0}\right)$, or $h_{0}=\widetilde{h}_{0}=e$,

$$
\omega^{*}=\frac{1}{2} \Omega\left(\left(h_{\pi} \widehat{g}_{+}, h_{\pi} \widehat{g}_{-}\right)\right) .
$$

Points of the phase space reduced by gauge symmetries $P^{*}$, can be described by pairs $\left([h],\left(\widehat{g}_{+}, \widehat{g}_{-}\right)\right)$where by $[h]$ we denote a homotopy class of maps $h:[0, \pi] \rightarrow G$ with fixed boundary values $h(0)=e, h(\pi)=h_{\pi}$ and such that $\left(h(x) \widehat{g}_{+}, h(x) \widehat{g}_{-}\right) \in D_{0}$. The symplectic form on $P^{*}$ can be described as the pullback of $\Omega$ by the map $\left([h],\left(\widehat{g}_{+}, \widehat{g}_{-}\right)\right) \mapsto\left(h_{\pi} \widehat{g}_{+}, h_{\pi} \widehat{g}_{-}\right)$.

Note the duality between $P$ and $P^{*}$. The symplectic forms of the two models coincide upon the exchange of $h_{\pi}$ with $\widehat{g}^{-1}$ and $\left(\widehat{g}_{+}, \widehat{g}_{-}\right)$with $\left(h_{\pi+}^{-1}, h_{\pi-}^{-1}\right)$. Variables $\left(h_{\pi+}, h_{\pi-}\right)$ in the $G$ model or $h_{\pi}$ in the $G^{*}$ model are associated to boundary values of their corresponding fields (this can be more explicitly seen working in a manifold $\Sigma$ whose boundary has more than two connected components) while $\widehat{g}$ and $\left(\widehat{g}_{+}, \widehat{g}_{-}\right)$are associated to the bulk. In this sense one can say that the duality previously described between the Poisson-Lie Sigma models for $G$ and $G^{*}$ can be stated as a bulk-boundary duality.

\section{More general Poisson Sigma models on $G$}

In this section we solve the model with Poisson structure (3.10) following the lines of section 4. As remarked in section 3 this structure does not make $G$ a Poisson Lie group, but indeed the left and right product by the corresponding Poisson-Lie groups are Poisson actions. In the resolution of the model we shall introduce a generalization of the dressing transformation and of the Heissenberg double and we shall be able to identify the symplectic leaves for the Poissson structure defined on $G$.

The action for the model is,

$$
S(g, A)=\int_{\Sigma} \operatorname{tr}\left(d g g^{-1} \wedge A\right)-\frac{1}{4} \operatorname{tr}\left(A \wedge\left(r+A d_{g} r^{\prime} A d_{g}^{-1}\right) A\right)
$$

where as stressed in section $3 r$ and $r^{\prime}$ are two solutions of the modified Yang-Baxter equation (3.5) with the same value for $\alpha$. The equations of motion are

$$
\begin{aligned}
d g g^{-1}+\frac{1}{2}\left(r+A d_{g} r^{\prime} A d_{g}^{-1}\right) A & =0 \\
d \widetilde{A}-[\widetilde{A}, \widetilde{A}]_{r^{\prime}} & =0, \quad \widetilde{A}:=A d_{g}^{-1} A
\end{aligned}
$$

From the previous equations, or performing in the action variations of the fields that keep $\widetilde{A}$ unchanged, we obtain

$$
d A+[A, A]_{r}=0
$$


The gauge symmetry in its infinitesimal form is

$$
\begin{aligned}
\delta_{\beta} g g^{-1}= & -\frac{1}{2}\left(r+A d_{g} r^{\prime} A d_{g}^{-1}\right) \beta \\
\delta_{\beta} A= & d \beta+[A, \beta]_{r}-\frac{1}{2}\left[d g g^{-1}+\frac{1}{2}\left(r+A d_{g} r^{\prime} A d_{g}^{-1}\right) A, \beta\right] \\
& \beta: \Sigma \rightarrow \mathfrak{g}
\end{aligned}
$$

The transformation for $g$ corresponds to the right dressing vector field that comes, like in the Poisson-Lie case of section 4 , from the contraction of the Poisson structure with the right-invariant forms. On-shell, $\left[\delta_{\beta_{1}}, \delta_{\beta_{2}}\right]=\delta_{\left[\beta_{1}, \beta_{2}\right]_{r}}$. Hence, the symmetry group is the one corresponding to the matrix $r$, i.e. $G_{r}$. The reason for the preferred role of $r$ against $r^{\prime}$ in the gauge symmetry is simply the choice of right-invariant one forms to express the Poisson structure. Had we chosen left-invariant forms (i.e. changing the variable $A$ by $\widetilde{A}$ in the action) the symmetry algebra would have been that of $r^{\prime}$.

As before we shall take $\alpha=1$. Then we can consider $G_{r}$ or $G_{r^{\prime}}$ as different subgroups of the same double group $D$. We shall denote by $\left(h_{+}, h_{-}\right)\left(\right.$resp. $\left.\left(h_{+^{\prime}}, h_{-^{\prime}}\right)\right)$ the elements of $G_{r}$ (resp. $\left.G_{r^{\prime}}\right)$.

Using the methods of section 4 , we can easily solve the model. As before, we first write locally the solutions for $A$ and $\widetilde{A}$,

$$
\begin{aligned}
A= & h_{+} d h_{+}^{-1}-h_{-} d h_{-}^{-1} \\
\widetilde{A}= & \widetilde{h}_{-^{\prime}} d \widetilde{h}_{-^{\prime}}^{-1}-\widetilde{h}_{+^{\prime}} \widetilde{h}_{+^{\prime}}^{-1} \\
& \left(h_{+}, h_{-}\right): U \rightarrow G_{r}, \quad\left(\widetilde{h}_{+^{\prime}}, \widetilde{h}_{-^{\prime}}\right): U \rightarrow G_{r^{\prime}}, \quad \Sigma \supset U \text { open contract. }
\end{aligned}
$$

with $h_{ \pm}\left(\sigma_{0}\right)=\widetilde{h}_{ \pm^{\prime}}\left(\sigma_{0}\right)=e$

The equation of motion for $g$ eq. (6.2) can be transformed into

$$
d g g^{-1}+r_{ \pm} A+A d_{g} r_{\mp}^{\prime} \widetilde{A}=0
$$

and inserting the solutions for $A, \widetilde{A}$

$$
d g g^{-1}+h_{ \pm} d h_{ \pm}^{-1}-A d_{g} \widetilde{h}_{\mp^{\prime}} d \widetilde{h}_{\mp^{\prime}}^{-1}=0
$$

Or equivalently

$$
h_{+}^{-1} g \widetilde{h}_{-^{\prime}}=h_{-}^{-1} g \widetilde{h}_{+^{\prime}}=\widehat{g}
$$

with $\widehat{g}=g\left(\sigma_{0}\right) \in G$.

We can write the general solution as an equation in $D$ for $g(\sigma)$,

$$
\left(g(\sigma) \widetilde{h}_{-^{\prime}}(\sigma), g(\sigma) \widetilde{h}_{+^{\prime}}(\sigma)\right)=\left(h_{+}(\sigma) \widehat{g}, h_{-}(\sigma) \widehat{g}\right) .
$$

If we now define $D_{0}^{\prime}:=G_{r} G_{d} \cap G_{d} G_{-r^{\prime}}$, we see that for solutions of the equations of motion $\left(h_{+}(\sigma) \widehat{g}, h_{-}(\sigma) \widehat{g}\right) \in D_{0}^{\prime}$. The symplectic leaves of $G_{r, r^{\prime}}$ are connected components of the orbits of the generalized dressing transformation of $\widehat{g}$ by $\left(h_{+}, h_{-}\right) \in G_{r}$, that comes from solving eq. (6.6) in $g(\sigma)$. 
In order to describe the presymplectic structure in the space of solutions we need to introduce a new Poisson bracket in $D$. Recall that the Heisenberg double was defined in section 3 as $D_{R, R}$ with $R=P_{d}-P_{r}$. We can generalize this construction by introducing another $r$-matrix $r^{\prime}$ that give rise to $R^{\prime}=P_{d}-P_{-r^{\prime}}$. The Poisson structure in the double we are interested in is $D_{R, R^{\prime}}$ that is again non-degenerate around the unit. Its main symplectic leaf is $D_{0}^{\prime}$. If we parametrize the points in $D_{0}^{\prime}$ by $\left(\eta_{+} \xi, \eta_{-} \xi\right)=\left(\widetilde{\xi} \widetilde{\eta}_{-^{\prime}}, \widetilde{\xi} \widetilde{\eta}_{+^{\prime}}\right)$, the symplectic structure in $D_{0}^{\prime}$ obtained by inverting the Poisson bracket is

$$
\Omega^{\prime}\left(\eta_{+} \xi, \eta_{-} \xi\right)=\operatorname{tr}\left[d \widetilde{\xi}^{-1} \wedge\left(d \eta_{+} \eta_{+}^{-1}-d \eta_{-} \eta_{-}^{-1}\right)-\xi^{-1} d \xi \wedge\left(\widetilde{\eta}_{+^{\prime}}^{-1} d \widetilde{\eta}_{+^{\prime}}-\widetilde{\eta}_{-^{\prime}}^{-1} d \widetilde{\eta}_{-^{\prime}}\right)\right] .
$$

Note again that although for a given point of $D_{0}^{\prime}$ factors $\xi, \eta_{ \pm}, \widetilde{\xi}, \widetilde{\eta}_{ \pm^{\prime}}$ in general are not uniquely determined (different choices differ by elements of the discrete groups $G_{0}$ or $G_{0}^{\prime}$ ) the form $\Omega^{\prime}$ is not affected by the ambiguity and is indeed well defined in $D_{0}^{\prime}$.

The presymplectic structure of the $r, r^{\prime}$ Poisson-Sigma model $\omega^{\prime}$ in the open geometry $(\Sigma=[0, \pi] \times \mathbb{R})$ with the boundary conditions of section 4 can then be written in terms of $\Omega^{\prime}$. It reads

$$
\omega^{\prime}=\Omega^{\prime}\left(\left(h_{\pi+} \widehat{g}, h_{\pi-} \widehat{g}\right)\right)-\Omega^{\prime}\left(\left(h_{0+} \widehat{g}, h_{0-} \widehat{g}\right)\right)
$$

with $h_{0 \pm}=h_{ \pm}(0) h_{\pi \pm}=h_{ \pm}(\pi)$. And if we take $\sigma_{0}=\left(0, t_{0}\right)$, i.e. $h_{0 \pm}=e$,

$$
\omega^{\prime}=\Omega^{\prime}\left(\left(h_{\pi+} \widehat{g}, h_{\pi-} \widehat{g}\right)\right) .
$$

The discussion of the gauge transformations and the reduced phase space goes parallel to the previous models. The points of the reduced phase space $P^{\prime}$ are pairs $\left(\left[\left(h_{+}, h_{-}\right)\right], \widehat{g}\right)$ with $\left[h_{+}, h_{-}\right]$the homotopy class of maps $[0, \pi] \rightarrow G_{r}$ with fixed boundary values and such that $\left(h_{+}(x) \widehat{g}, h_{-}(x) \widehat{g}\right) \in D_{0}^{\prime}$. The symplectic form in $P^{\prime}$ can be obtained as the pullback of $\Omega^{\prime}$ by the $\operatorname{map}\left(\left[\left(h_{+}, h_{-}\right)\right], \widehat{g}\right) \mapsto\left(h_{\pi+} \widehat{g}, h_{\pi-} \widehat{g}\right) \in D_{0}^{\prime}$.

\section{Conclusions}

We have solved the Poisson Sigma model for a Poisson-Lie group $G$ and for its dual group $G^{*}$. This models have non-regular foliations. For example, as both are Poisson-Lie groups, the unit of the respective groups forms itself a symplectic leaf. Due to this fact they fall out of the general construction of solutions of [6] and, as we have the explicit solutions at hand, they are an interesting example in which we can test the methods of [6] for the case of non regular leaves. Actually in many aspects our models can be taken as a deformation of the linear one.

Another remarkable aspect of the models under consideration is their duality. We can take $G$ and $G^{*}$ as subgroups of the same double group $D=G \times G, G_{d}$ and $G_{r}$ respectively. In the resolution of the two models on the strip $[0, \pi] \times \mathbb{R}$, we find a close relation between their reduced phase spaces (obtained by taking quotient by the gauge symmetry). Actually we see that there exist maps from these spaces to $D_{0}$ such that their symplectic forms are obtained as the pullback by these maps of the symplectic form on the main symplectic leaf of the Heisenberg double. This study reveals a bulk-boundary duality, such that the 
degrees of freedom naturally associated to the bulk in one model are mapped into those corresponding to the boundary in the other one. This suggests a connection with the Poisson-Lie non-abelian T-duality of [2] for the case of WZW models. The concrete form of this correspondence is not clear to us.

We have also solved the model on $G$ for the more general Poisson structure associated to a pair of $r$ matrices introduced in [17]. The solution is obtained along similar lines to those of the Poisson-Lie case. Now the symplectic form in the reduced phase space is obtained from a new Poisson structure in the double that generalizes the Heisenberg double of [17, 18, 3].

Throughout the paper we have considered $r$ - matrices that are solutions of the modified Yang-Baxter equation (3.5) with $\alpha=1$, i.e. factorizable Lie bialgebras of ref. 18]. In this case we can take the same double group $D=G \times G$ independently of the choice of $r$ because only $G_{r}$, the embedding of $G^{*}$ in $D$, depends on it. This allows us to carry out the study of the general case for a pair of $r$-matrices in section 6. The construction can be generalized without difficulties if $\alpha \neq 0$, but for the case $\alpha=0$ our methods have to be modified and the explicit form of the solutions requires further work. Despite this fact we would expect that the main results about duality remain essentially unchanged.

For instance, for the trivial model of eq. (4.1) with $r=0$ and its dual, the linear one of eq. (2.7), the reduced phase space is $T^{*}(G) \cong G \times \mathfrak{g}^{*} \cong G \times \mathfrak{g}$. In the trivial model the bulk degrees of freedom are in $G$ and those of the boundary in $\mathfrak{g}$, whereas for the linear model the situation is the converse. The symplectic form in both cases is the canonical form in $T^{*}(G)$.

As extensions of the work carried out in the paper one might consider more general geometries and boundary conditions. Another point that is worth mentioning is that of possible non-topological deformations of our models. In order to keep the gauge symmetry of the model one usually considers the addition of Cassimir functions on the Poisson manifold. One of these deformations, for the linear model, gives rise to the two dimensional Yang-Mills theory. It would be interesting to consider a generalizations of the latter in the context of Poisson-Lie groups.

An aspect that we do not cover in this paper is that of quantization of the models. The cases for Poisson-Lie groups have been extensively studied in the literature; see for instance 18, 10, 团. However, quantization of the generalized Heisenberg double $D_{R, R^{\prime}}$ of section 6 is much less known. It will be the subject of further research.

\section{References}

[1] A.Y. Alekseev and L.D. Faddeev, $\left(T^{*} G\right)_{t}$ : a toy model for conformal field theory, Commun. Math. Phys. 141 (1991) 413.

[2] A.Y. Alekseev, C. Klimčík and A.A. Tseytlin, Quantum Poisson-Lie T-duality and WZNW model, Nucl. Phys. B 458 (1996) 430 hep-th/9509123.

[3] A.Y. Alekseev and A.Z. Malkin, Symplectic structures associated to Lie-Poisson groups, Commun. Math. Phys. 162 (1994) 147 hep-th/9303038. 
[4] A.Y. Alekseev, P. Schaller and T. Strobl, The topological $G / G W Z W$ model in the generalized momentum representation, Phys. Rev. D 52 (1995) 7146 hep-th/9505012.

[5] L. Bergamin and W. Kummer, Graded Poisson sigma models and dilaton-deformed 2D supergravity algebra, J. High Energy Phys. 05 (2003) 074 hep-th/0209209]; The complete solution of $2 D$ superfield supergravity from graded Poisson-Sigma models and the super pointparticle, hep-th/0306217.

[6] M. Bojowald and T. Strobl, Classical solutions for Poisson Sigma models on a Riemann surface, J. High Energy Phys. 07 (2003) 002 hep-th/0304252.

[7] A.S. Cattaneo and G. Felder, A path integral approach to the Kontsevich quantization formula, Commun. Math. Phys. 212 (2000) 591 math.qa/9902090.

[8] A.S. Cattaneo, and G. Felder, Poisson Sigma models and symplectic groupoids, math.SG/0003023.

[9] M. Ertl, W. Kummer and T. Strobl, General two-dimensional supergravity from Poisson superalgebras, J. High Energy Phys. 01 (2001) 042 hep-th/0012219.

[10] F. Falceto and K. Gawędzki, Boundary G/G theory and topological Poisson-Lie Sigma model, Lett. Math. Phys. 59 (2002) 61 hep-th/0108206.

[11] F. Falceto and K. Gawędzki, Lattice Wess-Zumino-Witten model and quantum groups, J. Geom. Phys. 11 (1993) 251 hep-th/9209076.

[12] M. Hennaux and C. Teitelboim, Quantization of gauge systems. Princeton University Press, New Jersey, 1992.

[13] N. Ikeda, Two-dimensional gravity and nonlinear gauge theory, Ann. Phys. (NY) 235 (1994) 435 hep-th/9312059.

[14] M. Kontsevich, Deformation quantization of Poisson manifolds, I, IHES M97/72 preprint q-alg/9709040.

[15] J. Lu and A. Weinstein, Poisson-Lie groups, dressing transformations and Bruhat decompositions, J. Diff. Geom. 31 (1990) 501.

[16] P. Schaller and T. Strobl, Poisson structure induced (topological) field theories, Mod. Phys. Lett. A 9 (1994) 3129 hep-th/9405110.

[17] M.A. Semenov-Tian-Shansky, Dressing transformations and Poisson Group actions, Publ. RIMS 21 (1985) 1237.

[18] M.A. Semenov-Tian-Shansky, Poisson-Lie-Groups, quantum duality principle and the quantum double, Theor. Math. Phys. 93 (1992) 1292. 